\title{
Adaptive Neuro-Fuzzy Inference System based control of six DOF robot manipulator
}

\author{
Srinivasan Alavandar * and M. J. Nigam
}

Department of Electronics \& Computer Engineering, Indian Institute of Technology Roorkee, India-247667

\begin{abstract}
The dynamics of robot manipulators are highly nonlinear with strong couplings existing between joints and are frequently subjected to structured and unstructured uncertainties. Fuzzy Logic Controller can very well describe the desired system behavior with simple "if-then" relations owing the designer to derive "if-then" rules manually by trial and error. On the other hand, Neural Networks perform function approximation of a system but cannot interpret the solution obtained neither check if its solution is plausible. The two approaches are complementary. Combining them, Neural Networks will allow learning capability while Fuzzy-Logic will bring knowledge representation (Neuro-Fuzzy). This paper presents the control of six degrees of freedom robot arm (PUMA Robot) using Adaptive Neuro Fuzzy Inference System (ANFIS) based PD plus I controller. Numerical simulation using the dynamic model of six DOF robot arm shows the effectiveness of the approach in trajectory tracking problems. Comparative evaluation with respect to PID, Fuzzy PD+I controls are presented to validate the controller design. The results presented emphasize that a satisfactory tracking precision could be achieved using ANFIS controller than PID and Fuzzy PD+I controllers
\end{abstract}

Keywords: ANFIS, Fuzzy PD+I, PUMA robot, Degree of Freedom (DOF), manipulator control..

\section{Introduction}

Industrial robot manipulators are mainly positioning and handling devices. The essential problem in controlling robots is to make the manipulator follow a desired trajectory. In general an N-degree of freedom (DOF) rigid robot manipulator is characterized by $\mathrm{N}$ nonlinear, dynamic, coupled differential equations [1-3]. The problem of controlling robot manipulators still offers many practical and theoretical challenges due to the complexities of the robot dynamics and the requirement to achieve high precision trajectory tracking in the cases of high-velocity movement and highly varying loads.

Conventional robot control methods depend heavily upon accurate mathematical modeling, analysis, and synthesis. These approaches are suitable for the control of robots that operate in structured environments. However, operations in unstructured environments require robots to perform much more complex tasks without an adequate analytical model. The most challenging problem in this field is that there are always

\footnotetext{
* E-mail address: seenu.phd@gmail.com

ISSN: 1791-2377 (C) 2008 Kavala Institute of Technology. All rights reserved.
}

uncertainties in the unstructured environments. These uncertainties are primarily due to sensor imprecision and unpredictability of the environment characteristics and its dynamics. [4] [5] [6].

On the other hand, the advent of fuzzy set techniques provides us with a powerful tool for solving demanding realworld problems with uncertain and unpredictable environments. Fuzzy controller can characterize better behavior comparing with classical linear PID controller because of its non linear characteristics [7]-[9]. Recently, fuzzy-logic and conventional-techniques have been combined (hybrid) to design FL controllers which pave to appropriate solution for controlling the robot manipulators [10-12].The research on the fuzzy controller has been focused on the twoinput fuzzy system [13].The general structure of the two-input fuzzy controller that uses 'error' and 'change in error' as the input variables [14].

With PD type controller, elimination of steady state error is not possible. And PI type has limitation in enhancing the transient response. However, three-input and one-output fuzzy system is too complex to construct the PID controller. It is very difficult to decide the fuzzy control rules intuitively. The fuzzy PID controllers that use multiple 'two-input fuzzy 
controller' or partially fuzzy modified linear PID controllers are emerging to overcome these problems. The fuzzy PID controllers that use multiple 'two-input fuzzy controller' or partially fuzzy modified linear PID controllers are emerging to overcome these problems [15-16].

Fuzzy PD+I Controller [16] suffers the difficulty of tuning a particular inference system to model a complex dynamical system based on the training data. It works well with imprecise dynamics or even with no knowledge about the model the system dynamics, but they do not have a learning capability of their own. Hence, in that work a learning mechanism is created if neural networks, which have good learning attributes, are hybridized with fuzzy systems.

Neuro-Fuzzy techniques [17] have emerged from the fusion of Neural Networks (ANN) and Fuzzy Inference Systems (FIS) and form a popular framework for solving real world problems. Jang et al., [18], propose an Adaptive Neuro Fuzzy Inference System, in which a polynomial is used as the defuzzifier. This structure is commonly referred to as ANF1S. ANFIS distinguishes itself from normal fuzzy logic systems by the adaptive parameters, i.e., both the premise and consequent parameters are adjustable. Recent studies [20-23] have shown that the use of a controller that utilizes neural networks and fuzzy logic can be attractive. Takagi and Sugeno change the defuzzification procedure where dynamic systems are introduced as defuzzification subsystems. The potential advantage of the method is that under certain constraints, the stability of the system can be studied.

In this paper, an Adaptive Neuro Fuzzy Inference System (ANFIS) based PD plus conventional I controller is applied to the dynamic model of six DOF robot arm presented. Two inputs had been used, error (e), change of error $(\dot{e})$ that will be fed to ANFIS controller while integral error $(\Delta e)$ will be used as conventional integral action. This is due to difficulty of designing the rules for the integral action. The proposed controller is mainly focused on enhancement of the transient response. The proportional signal and the derivative signal are dominant to decide the transient response. But the integral signal whose major roll is to eliminate the steady state error has fixed gain. The ANFIS structure with first order Sugeno model, Gaussian membership functions with product inference rule are used at the fuzzification level. Hybrid learning algorithm that combines least square method with gradient descent method is used to adjust the parameter of membership function. To validate the performance, a comparison with the fuzzy PD+I and the linear PID is performed under same tuning.

Organization of the paper is as follows. Section 2 introduces the six degrees of freedom robot arm Puma 560 and its dynamic model. Section 3 describes the scheme of the hybrid Adaptive Neuro Fuzzy Inference System (ANFIS) based PD plus conventional I controller. Section 4 provides numerical simulation results to demonstrate the effectiveness of this hybrid controller and comparative evaluation of the ANFIS control method with that of fuzzy PD+I and the conventional PID is performed. And Section 5 discusses the benefits of the studied adaptive neuro fuzzy control law and conclusions are presented.

\section{Dynamics of Six DOF Puma robot}

The dynamics of a serial n-link rigid robot can be written as,

$M(q) \ddot{q}+c(q, \dot{q})+g(q)=\tau$

where $q$ is the $\mathrm{n} \times 1$ vector of joint displacements, $\dot{q}$ is the $\mathrm{n}$ $\mathrm{x} 1$ vector of joint velocities, $\tau$ is the $\mathrm{n} \times 1$ vector of actuators applied torques, $M(q)$ is the $\mathrm{n} \times \mathrm{n}$ symmetric positive definite manipulator inertia matrix, $c(q, \dot{q})$ is the $\mathrm{n}$ $\mathrm{x} 1$ vector of centripetal and Coriolis torques and $g(q)$ is the $\mathrm{n} \times 1$ vector of gravitational torques obtained as the gradient of the potential energy $U(q)$ due to gravity. We assume the robot joints are joined together with revolute joints.

Let the desired joint position $q_{d}$ be a twice differentiable vector function. We define a control problem to determine the actuator torques in such a way that the following control aim be achieved.

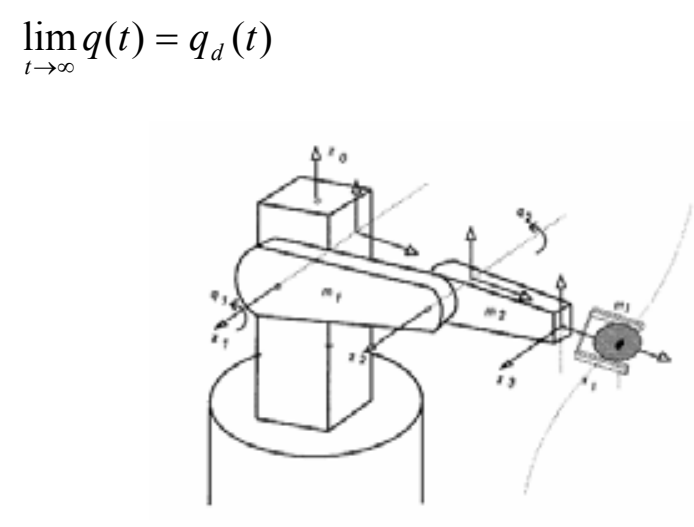

Fig. 1 PUMA robot

A six-degrees-of-freedom PUMA-560 robot is considered for the simulation, the kinematical and dynamical parameters of the arm are adopted from the work of Armstrong [19]. The electrical parameters of the motors are assumed by comparing the size and power of the PUMA motors with well-documented commercially-available DC motors, and then interpolating the corresponding parameters of interest are taken from our previous work [16]. Figure 1 shows the structure of PUMA robot. The overall block diagram of the system under control is shown in Figure 2.

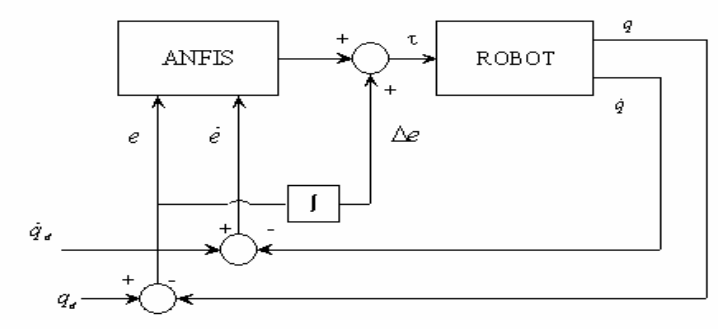

Fig. 2. The overall block diagram of the system 


\section{Adaptive Neuro Fuzzy Inference System (ANFIS based controller design}

This section introduces the basics of ANFIS network architecture and its hybrid learning rule. Inspired by the idea of basing the fuzzy logic inference procedure on a feedforward network structure, Jang [18] proposed a fuzzy neural network model - the Adaptive Neural Fuzzy Inference System or semantically equivalently, Adaptive Network-based Fuzzy Inference System (ANFIS), whose architecture is shown in Figure 3. He reported that the ANFIS architecture can be employed to model nonlinear functions, identify nonlinear components on-line in a control system, and predict a chaotic time series.

It is a hybrid neuro-fuzzy technique that brings learning capabilities of neural networks to fuzzy inference systems. The learning algorithm tunes the membership functions of a Sugeno-type Fuzzy Inference System using the training inputoutput data. The ANFIS is, from the topology point of view, an implementation of a representative fuzzy inference system using a BP neural network-like structure. It consists of five layers. The role of each layer is briefly presented as follows: let $O_{i}{ }^{l}$ denote the output of node $i$ in layer $l$, and $x_{i}$ is the $i^{\text {th }}$ input of the ANFIS, $i=1,2, \ldots, p$. In layer 1 , there is a node function $M$ associated with every node:

$$
O_{i}^{1}=M_{i}\left(x_{i}\right)
$$

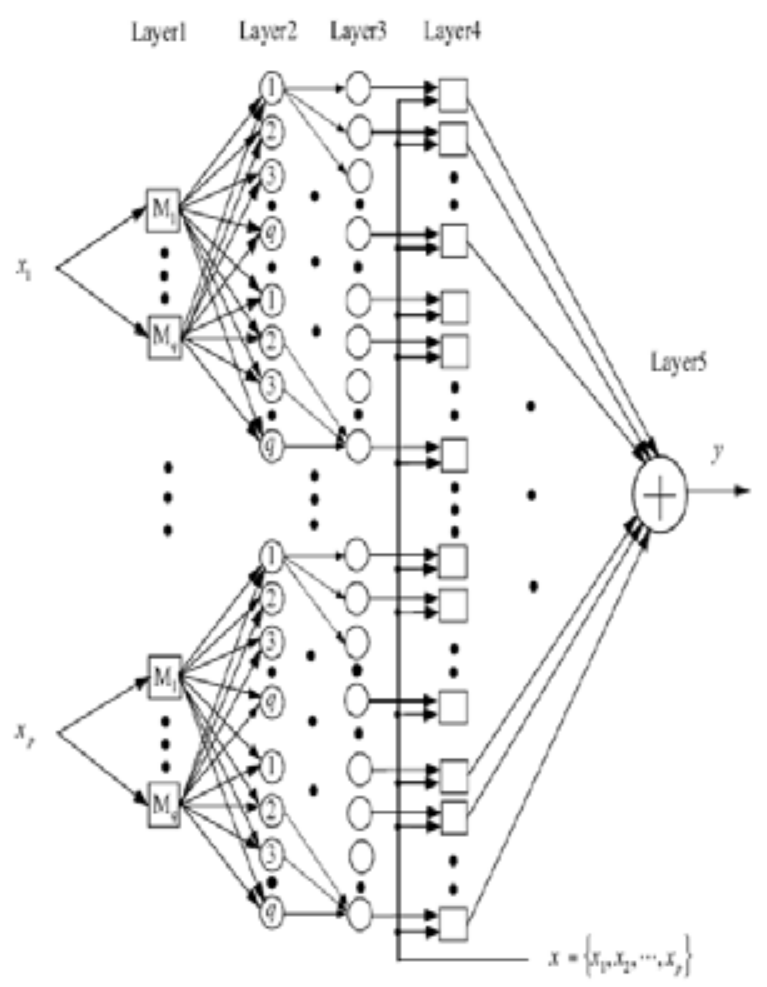

Fig. 3. Structure of ANFIS

The role of the node functions $M_{1}, M 2 \ldots$ Mq here is equal to that of the membership functions $\mu(x)$ used in the regular fuzzy systems, and $q$ is the number of nodes for each input.
Gaussian shape functions are the typical choices. The adjustable parameters that determine the positions and shapes of these node functions are referred to as the premise parameters. The output of every node in layer 2 is the product of all the incoming signals:

$$
O_{i}^{2}=M_{i}\left(x_{i}\right) A N D M_{j}\left(x_{j}\right)
$$

Each node output represents the firing strength of the reasoning rule. In layer 3 , each of these firing strengths of the rules is compared with the sum of all the firing strengths. Therefore, the normalized firing strengths are computed in this layer as:

$O_{i}^{3}=\frac{O_{i}^{2}}{\sum_{i} O_{i}{ }^{2}}$

Layer 4 implements the Sugeno-type inference system, i.e., a linear combination of the input variables of ANFIS, $x_{1}, x_{2}$, $\ldots x_{p}$ plus a constant term, $c_{1}, c_{2}, \ldots, c_{p}$, form the output of each IF -THEN rule. The output of the node is a weighted sum of these intermediate outputs:

$O_{i}^{4}=O_{i}^{3} \sum_{j=1}^{p} P_{j} x_{j}+c_{j}$

where parameters $P_{1}, P_{2}, \ldots, P_{p}$ and $\mathrm{c}_{1}, \mathrm{c}_{2}, \ldots, \mathrm{c}_{\mathrm{p}}$, in this layer are referred to as the consequent parameters. The node in layer 5 produces the sum of its inputs, i.e., defuzzification process of fuzzy system (using weighted average method) is obtained:

$$
O_{i}^{5}=\sum_{i} O_{i}^{4}
$$

The flowchart of ANFIS procedure is shown in Figure 4. ANFIS distinguishes itself from normal fuzzy logic systems by the adaptive parameters, i.e., both the premise and consequent parameters are adjustable. The most remarkable feature of the ANFIS is its hybrid learning algorithm. The adaptation process of the parameters of the ANFIS is divided into two steps. For the first step of the consequent parameters training, the Least Squares method (LS) is used, because the output of the ANFIS is a linear combination of the consequent parameters. The premise parameters are fixed at this step. After the consequent parameters have been adjusted, the approximation error is back-propagated through every layer to update the premise parameters as the second step. This part of the adaptation procedure is based on the gradient descent principle, which is the same as in the training of the BP neural network. The consequence parameters identified by the LS method are optimal in the sense of least squares under the condition that the premise parameters are fixed. 


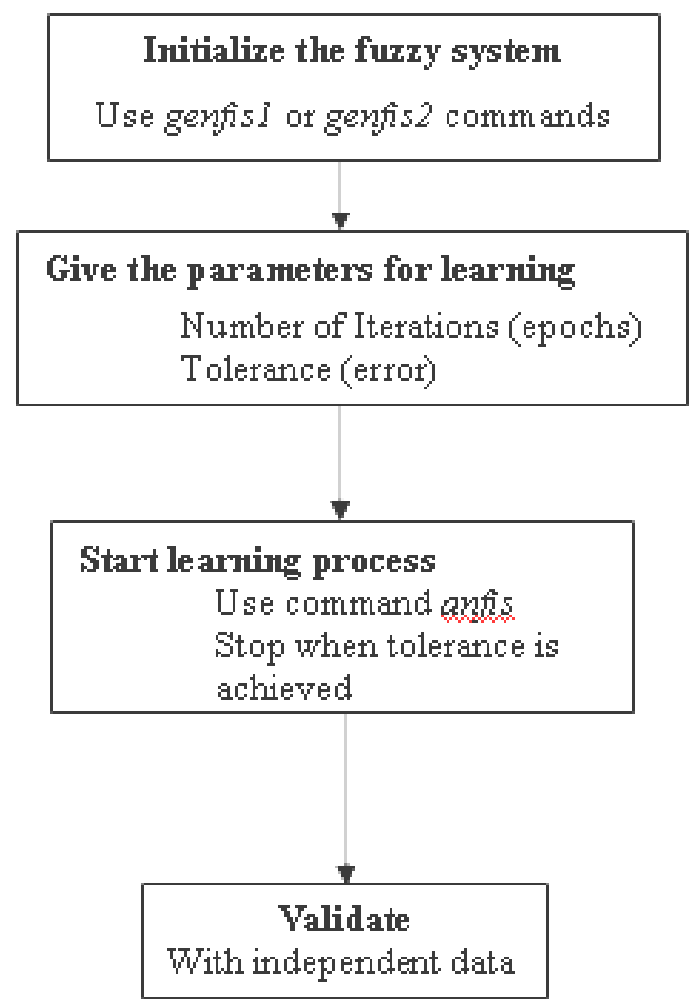

Fig. 4. ANFIS procedure

Therefore, this hybrid learning algorithm is more effective than the pure gradient decent approach, because it reduces the search space dimensions of the original back propagation method. The pure BP learning process could easily be trapped into local minima. When compared with employing either one of the above two methods individually, the ANFIS converges with a smaller number of iteration steps with this hybrid learning algorithm. This paper considers the ANFIS structure with first order Sugeno model containing 36 rules. Gaussian membership functions with product inference rule are used at the fuzzification level. Hybrid learning algorithm that combines least square method with gradient descent method is used to adjust the parameter of membership function.

In this structure, the Adaptive neuro fuzzy system is applied only to the proportional and derivative signal of the linear PID controller. The integral signal uses conventional linear method. The major roll of the integral signal is to eliminate the steady state error. The transient response is affected mostly by the proportional signal and the derivative signal. For the enhancement of the transient response, the varying gains are implemented on the proportional and derivative parts using two-input fuzzy system. The nonlinearities emphasize the proportional gain when the tracking error is relatively large and accelerates decreasing speed of the tracking error. The nonlinearities in the derivative gain suppress overshoot and increase damping from the beginning of the settling.

The control will take place in the joint space. Each joint has its own PID controller. The control action of the fuzzy controller was scaled to a torque control action applied to the PUMA dynamic model. The first priority is to tune the scaling factors, because these are the global tuning parameters that affect the overall control performance. In adjusting these, consideration is given to rise time $\left(t_{\mathrm{r}}\right)$, overshoot $\left(M_{P}\right)$ and the steady state error $\left(e_{s s)}\right.$. When the response is far away from the desired value, the input scaling factors $(S F)$ are adjusted to reduce the rise time, and is later readjusted to prevent overshoot as the response approaches the desired value. The output scaling factors is tuned to limit the FLC output to a reasonable value and to reduce the steady state error basic manual tuning procedure that can be used for the input and output scaling factors is given in Table 2 .

Table 2.

Tuning of Scaling factors of Fuzzy PD controller

\begin{tabular}{llll}
\hline Increase in $S F$ & Effect on $t_{r}$ & Effect on $M_{P}$ & Effect on $e_{s s}$ \\
\hline $\mathrm{K}_{\mathrm{P}}$ & Decrease & Increase & Decrease \\
$\mathrm{K}_{\mathrm{D}}$ & Increase & Decrease & Small change \\
$\mathrm{K}_{\mathrm{U}}$ & Decrease & Increase & Decrease \\
\hline
\end{tabular}

In the proposed structure, the adaptive neuro fuzzy system is normalized with respect to the maximum range of the signal. Using this characteristic, the simulation and comparison between the ANFIS based PD plus conventional I controller, Fuzzy PD conventional I controller and the linear PID is done.

\subsection{Conventional integral control}

The conventional integral control has the simple purpose of eliminating the steady state error and maintaining some control constant output when the system that is been controlled requires it.

\section{Simulation and Results}

In this section, the simulation was run under MATLAB 7.01 with Fuzzy Logic Toolbox 2.2 (R14SP1). To demonstrate the effectiveness of the proposed Adaptive Neuro Fuzzy based PD plus conventional I controller, the dynamic model of sixdegrees-of-freedom PUMA-560 robot is considered. Kinematical and dynamical parameters of the arm are adopted from the work of Armstrong [19] and the electrical parameters are taken from [16].

Numerical simulations of conventional PID controller, Fuzzy PID and Fuzzy PD+I is performed under same manual tuning procedure as shown in Table 2 for a six degree of freedom robot arm. The same values of PID control gains are used for conventional PID controller, FPD + I controller and Adaptive Neuro Fuzzy based PD plus conventional I controller to examine the performance. The tracking response of various controllers and error profile are shown in the Figure 7 to 12. 


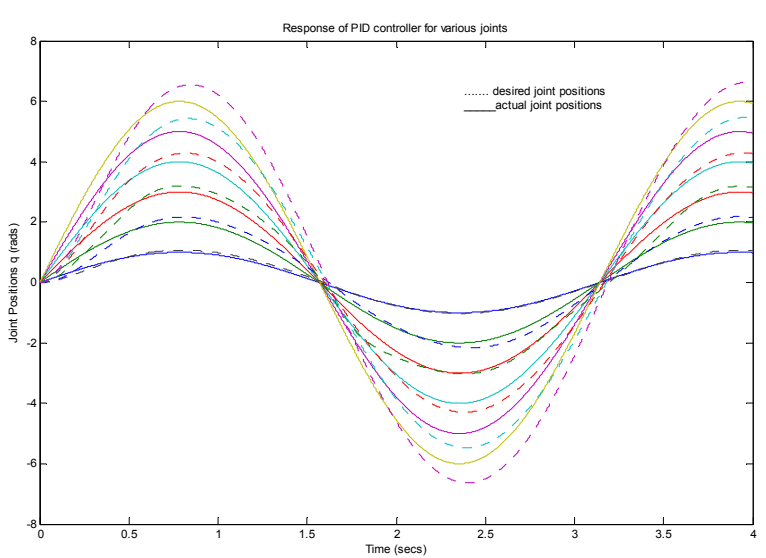

Fig. 7. Response of PID controller for various joints

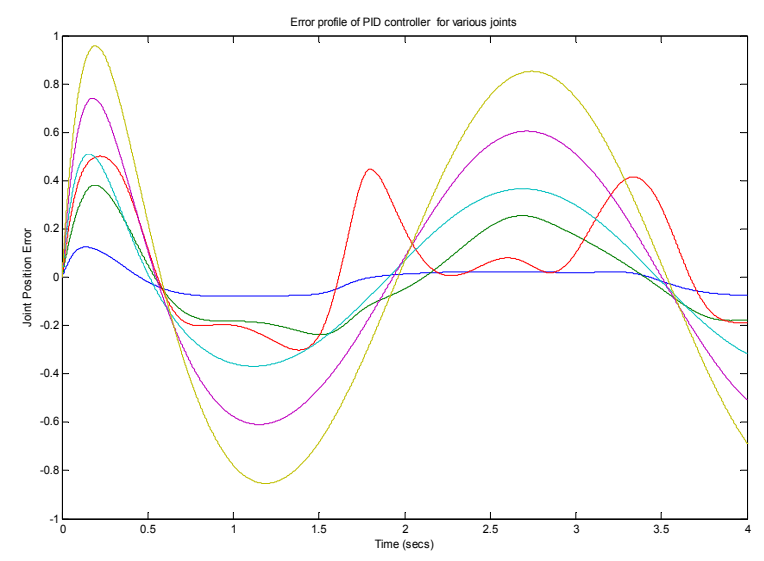

Fig. 8. Error profile of PID controller for various joints

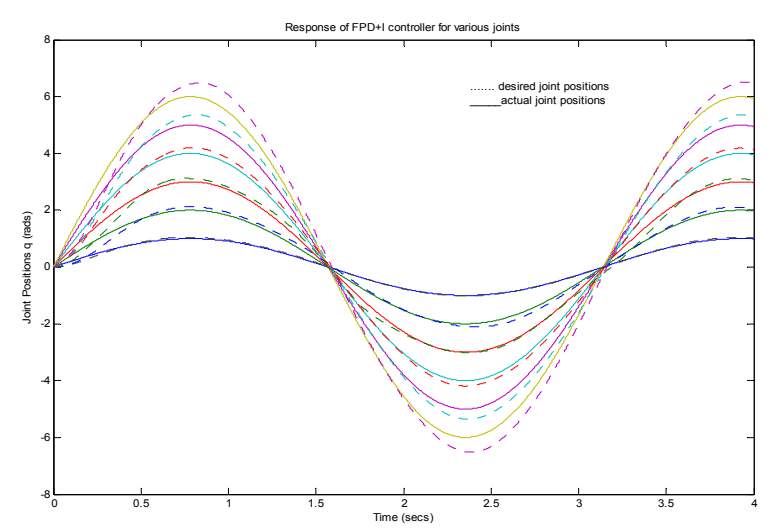

Fig. 9. Response of Fuzzy PD+I controller for various joints

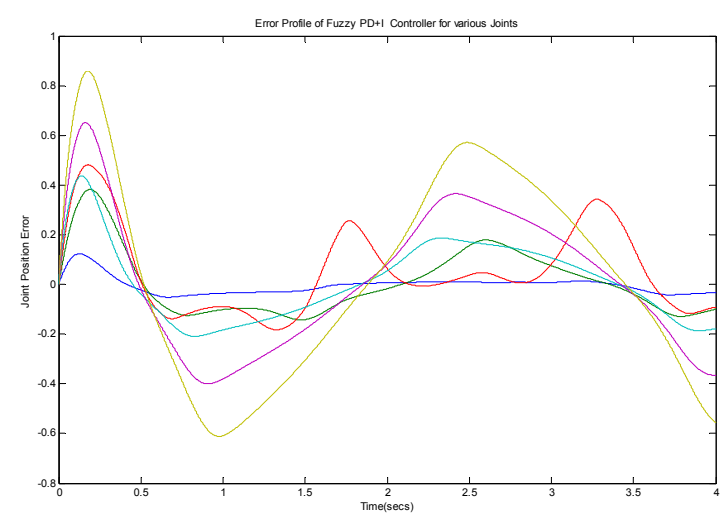

Fig. 10. Error profile of Fuzzy PD+I controller for various joints

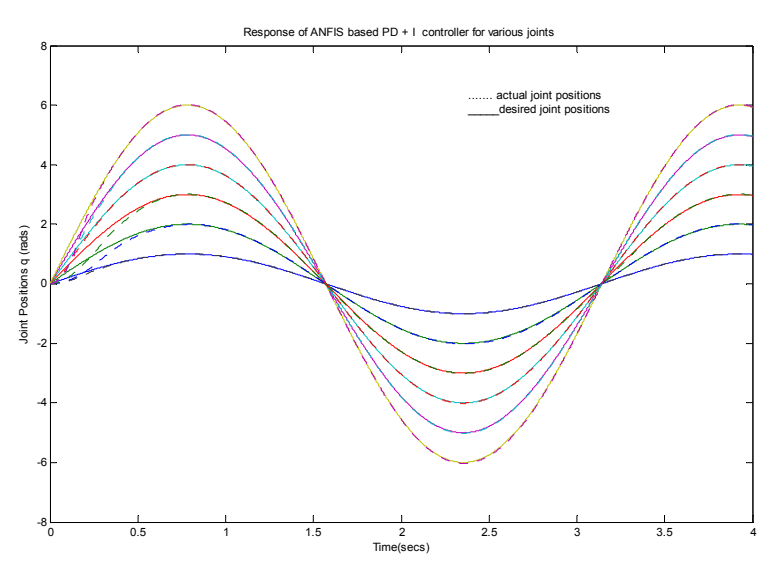

Fig. 11. Response of ANFIS based PD+I controller for various joints

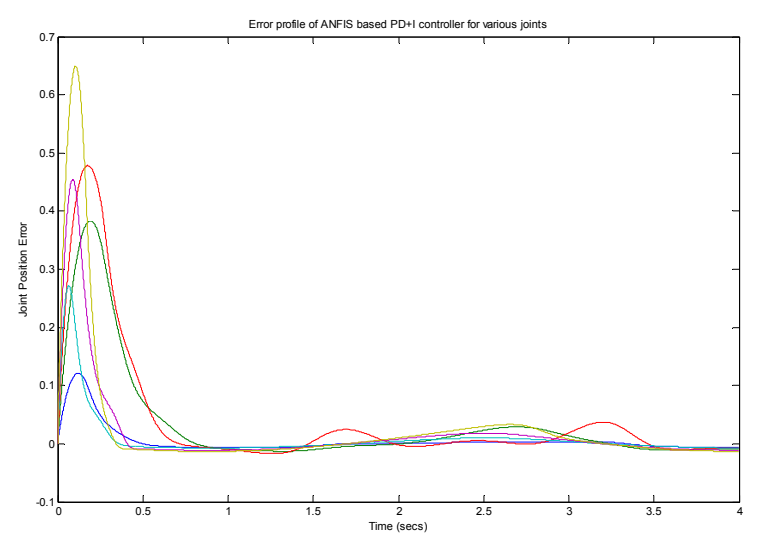

Fig. 12. Error profile of ANFIS based PD+I controller for various joints 


\section{Conclusions}

Due to the strong nonlinear characteristics and parameter variations in real environments, tracking control of a robot arm system is difficult. Proposed ANFIS based PD+I controller is mainly focused on enhancement of the transient response. Complexity of the three input fuzzy PID controller is minimized as possible and only two design variables are used to adjust the rate of variations of the proportional gain and derivative gain. Also the ANFIS converges with a smaller number of iteration steps with the hybrid learning algorithm. The ANFIS based PD $+\mathrm{I}$ controller developed in this paper provides a practical approach for such tasks. It is seen that the performance of ANFIS based PD+I controller improves appreciably compared to their respective fuzzy PD+I only or conventional PID counterparts. The results presented emphasize that a satisfactory tracking precision could be achieved using ANFIS based PD+I controller combination than fuzzy PD+I only or conventional PID only. Automatic tuning of the fuzzy system using neuro-fuzzy or genetic algorithm can be further studied.

\section{References}

1. Spong M, Vidyasagar M, (1989) Robot dynamics and control. Wiley, New York.

2. Craig JJ (1993) Adaptive control of manipulators through repeated trials. In: Spong MW, Lewis FL, Abdallah CT (eds) Robot control: dynamics, motion planning, and analysis. IEEE, IEEE Control Systems Society, pp 206-213

3. Craig JJ, Hsu P, Sastry SS (1987) Adaptive control of mechanical manipulators. Int Journal of Robotic Research 6(2):16-28

4. J. J. Craig, Introduction to Robotics: Mechanics and control, 2nd edition, Reading, A: Addison-Wesley, 1989

5. Lewis, F. L., Abdallah, C. T. and Dawson, D. M.: Control of Robot Manipulators. USA: Macmillan Publishing Company, a division of Macmillan, Inc., 1993.

6. O. Castillo, P. Melin, Intelligent adaptive model-based control of robotic dynamic systems with a new hybrid neuro-fuzzy-fractal approach, in: Proceedings of FLINS'2000, World Scientific, Bruges, Belgium, 2000, pp. 351-358

7. H. R. Berenji, "Fuzzy logic controllers," in An Introduction to Fuzzy Logic Application in Intelligent Systems, R. R. Yager and L. A. Zadeh, Eds. Boston, MA: Kluwer, 1992.

8. H.-J. Zimmermann, Fuzzy Sets Theory and Its Applications. Norwell, MA: Kluwer-Nijhoff, 1984.

9. E. H. Mamdani, "Application of fuzzy algorithm for control of simple dynamic plant," Proc. Inst. Elect. Eng., vol. 121, pp. 15851588,1974

10. Wei Li, "Design of a Hybrid Fuzzy Logic Proportional Plus Conventional

Integral-Derivative Controller", IEEE trans. on Fuzzy Systems, Vol. 6, No. 4. 1998, pp. 449-63.

11. Li, W. et al, "Tracking Control of a Manipulator under Uncertainty by FUZZY P + ID Controller”, Fuzzy Sets and Systems, vol. 122, 2001, pp. 125-137.

12. W. Li, X. G. Chang, J. Farrell, and F. M. Wahl, "Design of an enhanced hybrid fuzzy $p+i d$ controller for a mechanical manipulator," IEEE Transactions on Systems, Man, and Cybernetics-Part B: Cybernetics, vol. 31, December 2001.

13. G. K. I. Mann, B. G. Hu and R. G. Gosine, "Analysis of direct action fuzzy PID controller structures", IEEE Trans. Syst., Man, Cybern., vol. 29, no. 3, pp. 371-388, 1999.

14. H. Ying, "Theory and application of a novel fuzzy PID controller using a simplified Takagi-Sugeno rule scheme", Information Sciences, vol 123, pp. 281-293, 2000.

15. Misir, D., Malki, H. A. and Chen, G. 1996. Design and Analysis of a Fuzzy Proportional-integral- Derivative Controller, Fuzzy Sets and Systems, 79, 297-314.

16. Srinivasan Alavandar, M.J. Nigam Fuzzy PD+I Control of a Six DOF Robot Manipulator, Industrial Robot: An International Journal, vol. 35, issue 2, pp. 125-132, 2008.

17. Jang, J. S. R., C. T. Sun, E. Mizutani, Neuro-Fuzzy and Soft Computing, PTR Prentice Hall, 1997.

18. J. S. R. Jang, ANFIS: Adaptive-Network-based Fuzzy Inference Systems, IEEE Transactions on Systems, Man, and Cybernetics, Vol. 23, No. 03, pp. 665-685, 1993.
19. Brian Armstrong, Oussama Khatib, Joel Burdick, "The Explicit Dynamic Model and Inertial Parameters of the PUMA 560 Arm", Stanford Artificial Intelligence Laboratory Stanford University, IEEE Transactions and Systems 1986.

20. Tiberiu Vesselenyi, Simona Dzitac, Ioan Dzitac, Misu-Jan Manolescu, Fuzzy and Neural Controllers for a Pneumatic Actuator, International Journal of Computers, Communications and Control, Vol. 2, No. 4, pp. 375-387, 2007.

21. Y. L. Sun and M. J. Er, "Hybrid fuzzy control of robotics systems," IEEE Transactions on Fuzzy Systems, vol. 12, no. 6, pp. 755-765, 2004.

22. Hong-Rui Wang Li Yang Li-Xin We Fuzzy-neuro position/force control for robotic manipulators with uncertainties Soft Computing (2007) 11:311-315.

23. Duraisamy, N. Devarajan, D. Somasundareswari, A. Antony Maria Vasanth, S.N. Sivanandam, "Neuro fuzzy schemes for fault detection in power transformer", Applied Soft Computing 7 (2007) 534-539.

24. Peter I.corke, "The Unimation Puma Servo System", CSIRO Division of Manufacturing Technology, AUSTRALIA July 1994.

25. Peter I. Corke, "A Search for Consensus among Model Parameters Reported for the PUMA 560 Robot", CSIRO Division of Manufacturing Technology, Australia. Brain Armstrong-Helouvry University of Wisconsin Milwaukee, USA. July 1994. 